\title{
A 32-Channel Low-Intensity Focused Ultrasound Stimulator Using Phased Array Miniature Transducers for Enhanced Targeting Accuracy in Neuromuscular Rehabilitation System
}

\author{
Sungjin Oh, Hyungmin Kim, and Inchan Youn* \\ Center for Bionics, Korea Institute of Science and Technology, \\ 14-5 Hwarang-ro, Seongbuk-gu, Seoul 02792, Republic of Korea
}

(Received August 1, 2016; accepted December 19, 2016)

Keywords: ultrasound stimulator, phased array transducers, high targeting accuracy, low-intensity focused ultrasound, neuromuscular rehabilitation

Low-intensity pulsed ultrasound (LIPUS) stimulation can be used for noninvasive neuromuscular rehabilitation. For increasing stimulation efficiency, focused ultrasound (FUS) providing high-energy concentration should be used for LIPUS stimulation. However, because of difficulties in fine focus control, it is difficult to accurately target a stimulation site with conventional FUS stimulators using a single transducer. For implementing highly accurate targeting with a finely adjustable focal point, a 32-channel low-intensity focused ultrasound (LIFU) stimulator using phased array miniature piezoelectric transducers is presented in this paper. In the presented stimulator, a beamforming technology is used to delicately control the focal point without the difficulties in the conventional FUS stimulators. The experimental results show that the focal point was successfully adjusted in horizontal and vertical directions with nearly zero targeting errors. The ultrasound energy with the spatial peak-pulse average intensity $\left(I_{S P P A}\right)$ of 0.48 $\mathrm{W} / \mathrm{cm}^{2}$ was concentrated in a focusing area of $1.05 \mathrm{~mm}$ radius, which is sufficiently small to target peripheral nerves or a bundle of muscle fibers.

\section{Introduction}

Electrical stimulation is traditionally used for neuromuscular stimulation and rehabilitation. ${ }^{(1-3)}$ However, invasive surgical processes for electrical stimulation can cause infection that involves critical adverse effects on a patient's health. ${ }^{(3-5)}$ As an alternative stimulation method without surgery, ultrasound has been studied by many research groups..$^{(6-8)}$ Because ultrasound energy can be propagated to a target site without an implanted electrode, ultrasound can be effectively used for noninvasive neuromuscular stimulation and rehabilitation.

In particular, previous studies show that low-intensity pulsed ultrasound (LIPUS) stimulation has a positive effect on the regeneration of peripheral nerves and muscle tissues. ${ }^{(9-13)}$ In the previous works, LIPUS stimulation accelerated recovery of injured rat sciatic nerves, as compared with the sham group. ${ }^{(9,10)}$ In other studies, Schwann cell proliferation was enhanced by LIPUS stimulation. ${ }^{(11,12)}$ In addition, in clinical studies, LIPUS stimulation also increased the regeneration rate of inflammatory skeletal muscle tissues. ${ }^{(13,14)}$ 
For enhancing stimulation efficiency, ultrasound energy should be highly concentrated in a miniaturized target area. As compared with planar ultrasound that is generally used for imaging technologies, focused ultrasound (FUS) can concentrate a larger amount of energy in a smaller focusing area. Thus, it is desirable to utilize FUS in a LIPUS stimulation system for achieving high stimulation efficiency. In conventional FUS stimulation systems, a single FUS transducer with a fixed focus is used.(15-19) In this configuration, the transducer must be geometrically shifted for the planar control of the focal point. In addition, it is difficult to finely adjust a depth of focus (DOF) because it is only discretely controllable by replacing the transducer. Because of these limitations, it is difficult to accurately control the focal point in three dimensions using conventional FUS stimulators. To overcome these difficulties, it is necessary to develop a new FUS stimulation system where the focal point can be finely adjusted without the geometrical movement and inconvenient replacement of the transducer.

A beamforming method using multielement ultrasound transducers was presented to accurately control the focal point without the geometrical movement of the transducer. ${ }^{(20-22)}$ In the beamforming method, the focal position is determined according to the constructive interference of ultrasound waves. Thus, the location of the focal point can be adjusted as a function of time delays or phase differences, among the ultrasound waves that are generated from a transducer array. ${ }^{(20-22)}$ The time delays can be easily and finely controlled using an analog or digital time delay controller. ${ }^{(23,24)}$

In this paper, a 32-channel low-intensity focused ultrasound (LIFU) stimulator is presented for highly accurate targeting in neuromuscular rehabilitation. The presented stimulator generates the focused LIPUS to improve stimulation efficiency. Attributed to the beamforming technology, the focal point can be finely controlled using the presented system. For ultrasound beamforming, 32-channel phased array miniature piezoelectric (PZT) transducers were fabricated and used. Experiments to measure the spatial distributions of the output ultrasound energy in 2-D areas were conducted to evaluate the targeting accuracy of the developed system.

\section{Design and Fabrication}

\subsection{System architecture}

Figure 1 shows the system architecture of the presented 32-channel LIFU stimulator using the phased array miniature transducers. The stimulator is composed of two parts, a stimulator circuit and linear-type phased array miniature transducers with 32 elements. The stimulator circuit controls the parameters required for pulsed sonication, such as acoustic intensity, fundamental frequency, pulse repetition frequency, duty cycle, sonication duration, and interstimulus interval.(25) In addition, the stimulator circuit calculates the time delays for each transducer element as a function of the location of the target focal point. According to the calculated results, 32 time-delayed highvoltage bipolar pulses are generated in the stimulator circuit. Then, the linear-type phased array miniature transducers generate phase-shifted low-intensity ultrasound waves.

\subsection{Stimulator circuit}

A schematic of the developed LIFU stimulator circuit is depicted in Fig. 2. The stimulator circuit consists of a field-programmable gate array (FPGA)-based signal controller and a 32-channel high-voltage ultrasound pulser circuit. 

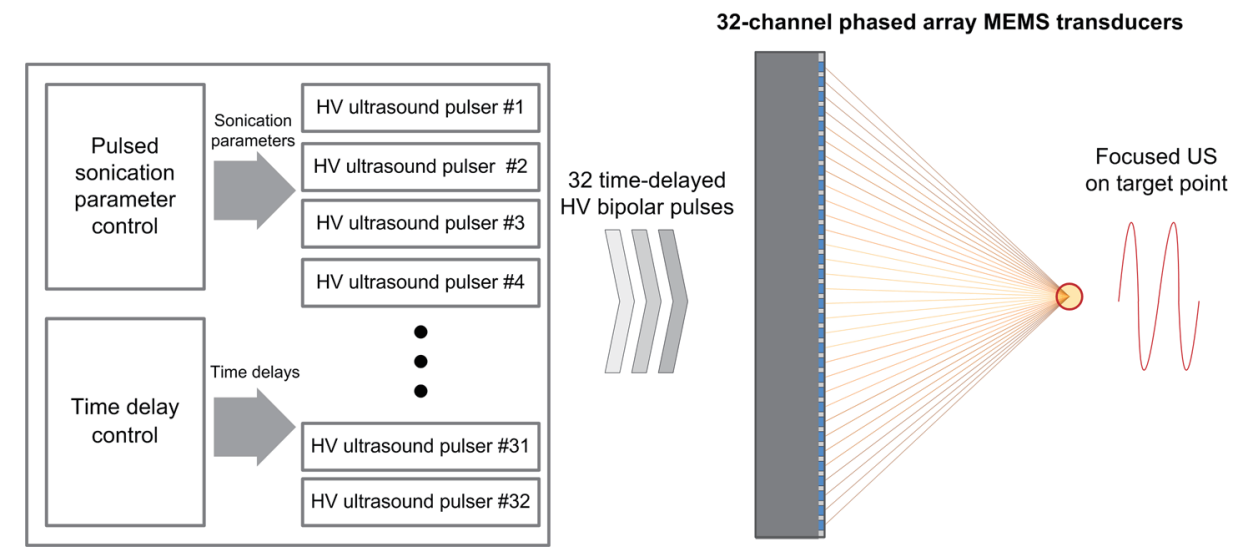

Fig. 1. (Color online) System architecture of the presented 32-channel LIFU stimulator using phased array MEMS transducers.

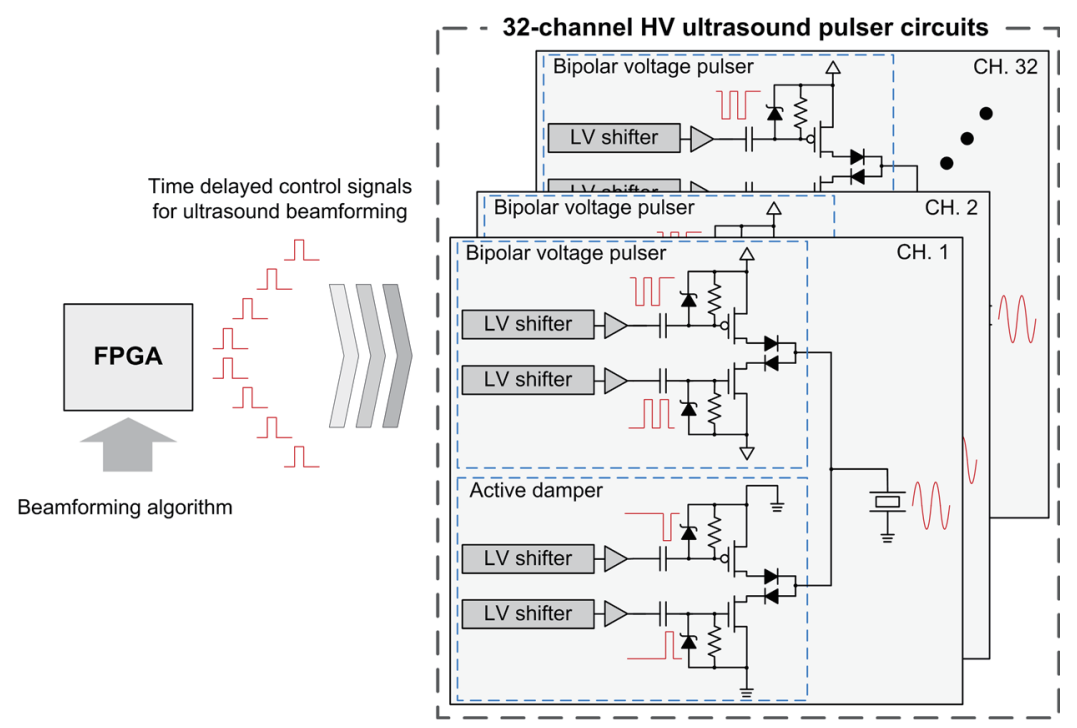

Fig. 2. (Color online) A schematic of the stimulator circuit that consists of a FPGA-based signal controller and 32-channel high-voltage ultrasound pulser circuits.

In the signal controller, the time delays for ultrasound beamforming are calculated according to the location of the target focal point. For time delay calculation, a focusing delay algorithm based on a wave speed and a propagation distance is used. ${ }^{(20-22)}$ The time delay for the ith transducer element is calculated as

$$
\tau_{i}=\frac{1}{c}\left[R_{f}-\sqrt{x_{f}^{2}+\left(y_{i}-y_{f}\right)^{2}}\right]
$$

where $c$ is the propagating speed of the ultrasound wave, $R_{f}$ is the maximum distance from the transducer array to the target focal point, $x_{f}$ is the horizontal position of the target focal point, $y_{i}$ is 
the vertical position of the ith transducer element, and $y_{f}$ is the vertical position of the target focal point. For enhancing calculation accuracy, an FPGA chip (Xilinx Spartan-6 XC6SLX16; Xilinx Inc., San Jose, CA) with a high clock frequency of $150 \mathrm{MHz}$ is used. This clock frequency can provide minimum focus shifting of $9.87 \mu \mathrm{m}$. According to the calculated time delays, the timedelayed control signals are generated and transmitted to the high-voltage ultrasound pulser circuits.

The high-voltage ultrasound pulser circuit is composed of a bipolar voltage pulser and an active damper. The former generates the time-delayed high-voltage bipolar pulses with the maximum peak-to-peak amplitude of $400 V_{P P}$. The latter is used to provide fast return-to-zero waveforms that reduce unwanted ripples after sonication. Level shifters (CD40109B; Texas Instruments Inc., Dallas, TX), high-voltage buffers (CD4503B; Texas Instruments Inc., Dallas, TX), zener clamps, and complementary pairs of power metal-oxide-semiconductor field-effect transistors (MOSFETs) (TC6320; Microchip Technology Inc., Chandler, AZ) are integrated in the bipolar pulsers and active damper circuits.

Figure 3 shows the fabricated 32-channel LIFU stimulator circuit. The stimulator circuit is integrated in a 6-layer printed circuit board (PCB) of $120 \times 80 \mathrm{~mm}^{2}$ size.

\subsection{Transducer array}

Cross-sectional diagrams of the 32-channel phased array miniature PZT transducers are shown in Fig. 4. At first, a PZT ceramic layer with a specific thickness to obtain the target resonance frequency was produced by the wet extrusion of multilayered PZT planes. Then, metal electrodes

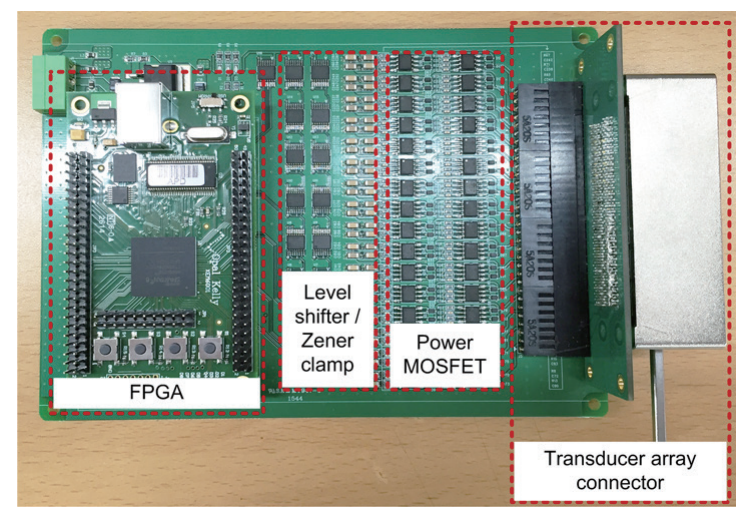

Fig. 3. (Color online) A fabricated stimulator circuit integrated in a $120 \times 80 \mathrm{~mm}^{2}$-sized 6-layer PCB.

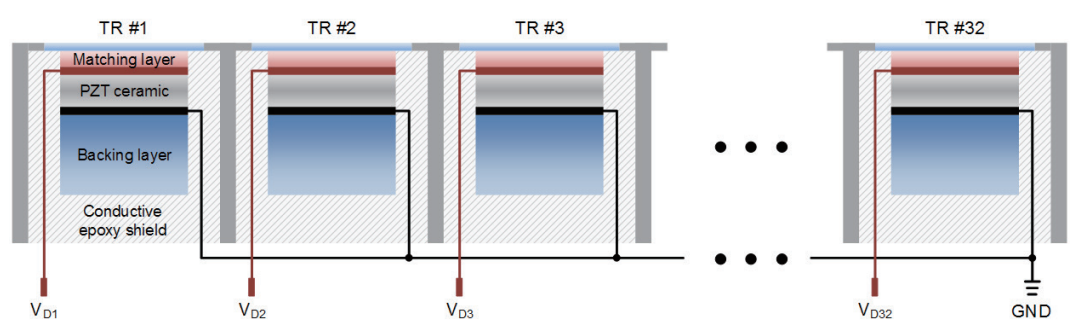

Fig. 4. (Color online) Cross-sectional diagrams of the 32-channel phased array PZT MEMS transducers used in the developed system. 
that are connected to the driving signal $\left(\mathrm{V}_{\mathrm{D} 1}-\mathrm{V}_{\mathrm{D} 32}\right)$ or ground signal (GND) were deposited on the fabricated PZT ceramic layer. An impedance matching layer was also attached in front of the PZT ceramic layer to improve sonication efficiency. Note that the impedance difference between the PZT ceramic and outer media should be minimized for highly efficient ultrasound pulsation. At the backside of the PZT ceramic layer, a backing layer was attached to absorb ultrasound waves that propagate to the backside of the transducer. The PZT ceramic layer, matching layer, and backing layer were covered with a conductive epoxy shield. Because the conductive epoxy shield provides an enlarged ground plane, transducer noise and other noises from the outside can be significantly reduced.

Figure 5 shows the fabricated miniature PZT transducer array with 32 elements that are connected to the stimulator circuit. The transducer array was custom fabricated by Digital ECHO Co., Ltd. (Hwaseong, Republic of Korea). The planar size of each transducer element is $12 \times 2$ $\mathrm{mm}^{2}$, and the center-to-center spacing between the transducers is $2.1 \mathrm{~mm}$. The size of the packaged transducer array is $20 \times 80 \times 19 \mathrm{~mm}^{3}$. The resonance frequency is set as $1.5 \mathrm{MHz}$ for all transducer elements.

\section{Experiments}

\subsection{Measurement of sonication performance}

For evaluating the sonication performance of the fabricated transducer and transducer driving functionality of the developed stimulator circuit, output performance measurement was conducted using a single transducer element in the fabricated transducer array. A hydrophone (HNR-0500; ONDA Corp., Sunnyvale, CA) was used to measure the ultrasound pressure waves from the transducer that was fixed in a water tank. The transducer element was driven by continuously repeated bipolar pulse trains with the peak-to-peak voltage amplitude of $60 V_{P P}$, fundamental frequency of $1.5 \mathrm{MHz}$, pulse repetition frequency of $1.5 \mathrm{kHz}$, and duty cycle of $50 \%$.

\subsection{Beamforming experiment}

A beamforming experiment was conducted to evaluate the energy focusing performance of the developed stimulator. An acoustic intensity measurement system (AIMS III; ONDA Corp., Sunnyvale, CA) and a real-time ultrasound energy plotting software (Soniq; ONDA Corp.,

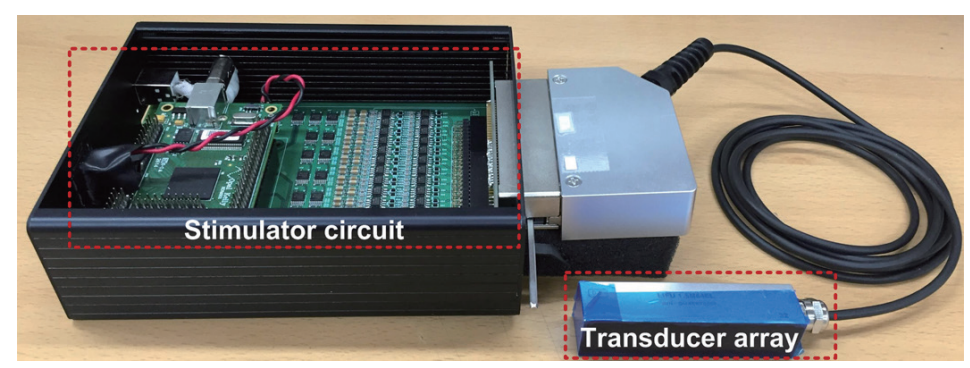

Fig. 5. (Color online) A fabricated 32-channel phased array PZT MEMS transducer assembled with the stimulator circuit. 
Sunnyvale, CA) were used to obtain the spatial distribution of the focused ultrasound energy. The fabricated miniature transducer array is fixed in a water tank, and a moving hydrophone measured the ultrasonic pressures. The measured ultrasound pressure waves were transmitted to an oscilloscope and PC, where the ultrasound energy intensity was calculated and plotted by the Soniq software program. The experimental environment is depicted in Fig. 6.

A geometrical setting of the experiment is depicted in Fig. 7. A total of 13 target focal points were designated in horizontal and vertical directions. The spacing between adjacent target focal points was fixed as $10 \mathrm{~mm}$ in both directions. All the target focal points in the horizontal direction were located with a DOF of $50 \mathrm{~mm}$. In the vertical direction, the target focal points were aligned at the center in the horizontal direction. The size of the ultrasound energy measured area was fixed at $80 \times 20 \mathrm{~mm}^{2}$ in both directions.

The developed LIFU stimulator generated beamformed ultrasound waves with varying focal positions. The transducer driving voltage was fixed at $60 V_{P P}$. The fundamental frequency, pulse repetition frequency, and duty cycle were fixed at $1.5 \mathrm{MHz}, 1.5 \mathrm{kHz}$, and $1 \%$, respectively. Note that the duty cycle of $1 \%$ was selected due to the requirement of the measuring system, AIMS III.

\section{Results}

\subsection{Sonication performance}

Figure 8 shows the results of the transducer driving performance test using the single transducer element in the fabricated transducer array. As plotted in Fig. 8(a), the transducer was successfully sonicated using the developed LIFU stimulator. The pulsed ultrasound waveforms were clearly generated without significant distortion such as a bias, frequency noises, and large variation of the amplitude that can be caused by reflected ultrasound waves or the initial overshoot of the driving signal. The spatial peak-pulse average intensity $\left(I_{S P P A}\right)$ and spatial peak-temporal average intensity

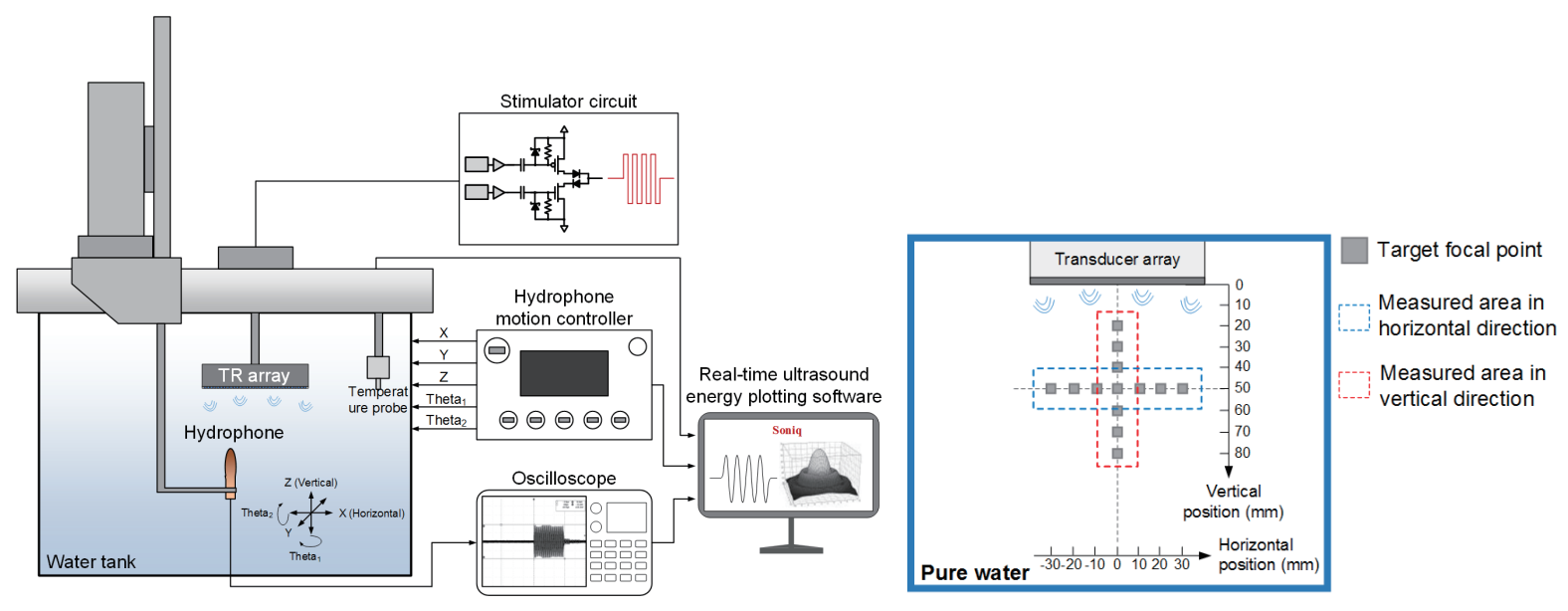

Fig. 6. (Color online) Experimental environment to measure spatial distributions of the beamformed ultrasound energy with varying focal points.
Fig. 7. (Color online) A geometrical setting used in the beamforming experiment. A total of 13 target focal points were assigned with $1.0 \mathrm{~mm}$ spacing in horizontal and vertical directions. 


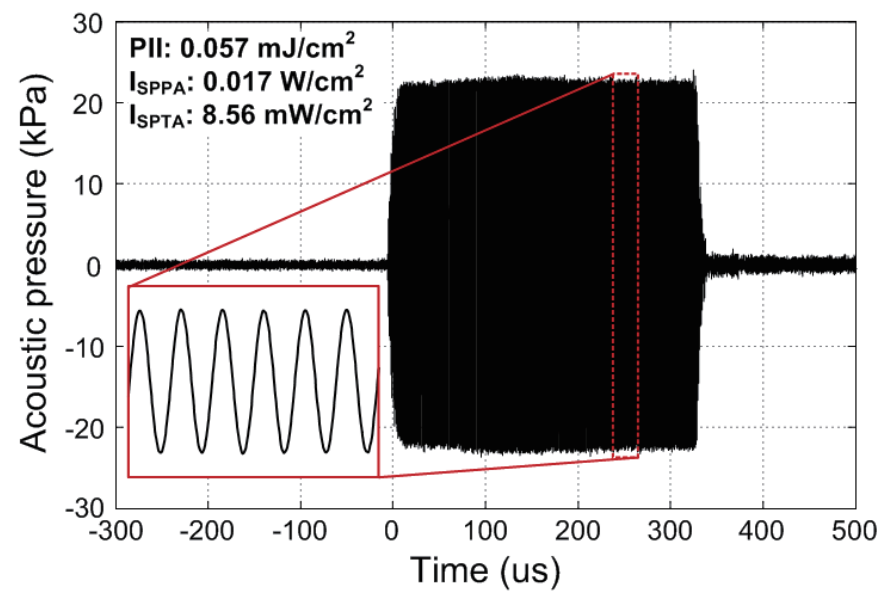

(a)

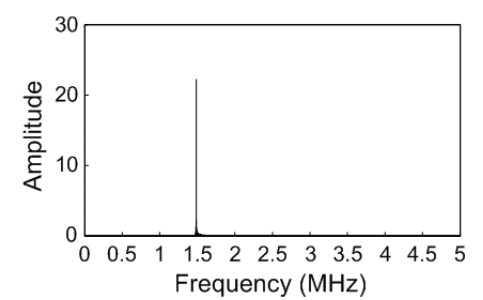

(b)

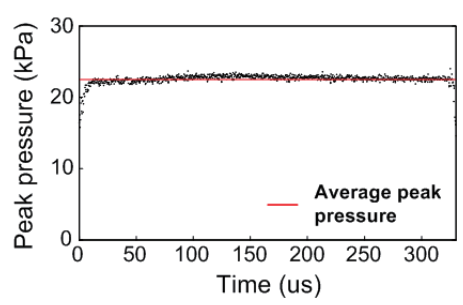

(c)

Fig. 8. (Color online) Results of the transducer driving performance test. (a) Measured ultrasound waveform and calculated acoustic energy. (b) FFT-based single-sided spectrum of the measured waveform. (c) Absolute peak pressure values of the sinusoidal waves in the measured waveform.

$\left(I_{S P T A}\right)$ were measured as 0.017 and $8.56 \mathrm{~mW} / \mathrm{cm}^{2}$, respectively. These energy intensities are $29.55 \%$ higher than those provided by a transducer made of a commercialized PZT ceramic layer (NCE51; Noliac, Kvistgard, Denmark).

For evaluating the degree of the signal distortion, a frequency domain analysis and an amplitude uniformity analysis were conducted. Figure $8(\mathrm{~b})$ shows the fast Fourier transform (FFT)-based single-sided spectrum. The fundamental frequency of the measured ultrasound waveform was measured as $1.49 \mathrm{MHz}$ with $0.67 \%$ error to the target frequency. In addition, there was no noticeable frequency noise. To evaluate the amplitude uniformity, the peak pressures of the sinusoidal waves in the measured ultrasound waveform were extracted and converted to the absolute values. In Fig. 8(c), the absolute values of the peak pressures are plotted as a function of time. The average and standard deviation of the absolute peak pressure values were calculated as 22.45 and $1.14 \mathrm{kPa}$, respectively, which indicate the small variation in the amplitude.

\subsection{Spatial distribution of focused ultrasound energy}

The spatial distributions of the focused ultrasound energy are plotted in Fig. 9. The pulse average intensity $\left(I_{P A}\right)$ is used to express the measured ultrasound energy. As shown in Fig. 9, it can be mentioned that the targeting of ultrasound energy was successfully conducted because, in all experimental sets, the $I_{S P P A}$ that indicates the maximum energy intensity in the measured area was observed at each target focal point. Although multifocal points with relatively low energy intensities were observed at several positions out of the target focal points, this was the natural phenomenon caused by the constructive interference of the ultrasound waves. In the horizontal direction, a zero targeting error was obtained. On the other hand, the targeting error was measured 


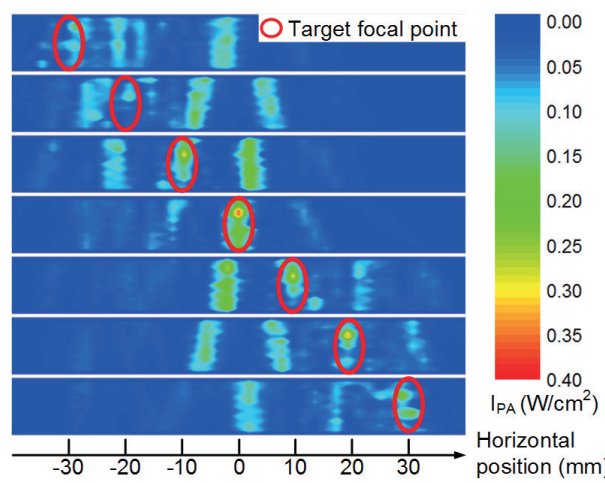

(a)
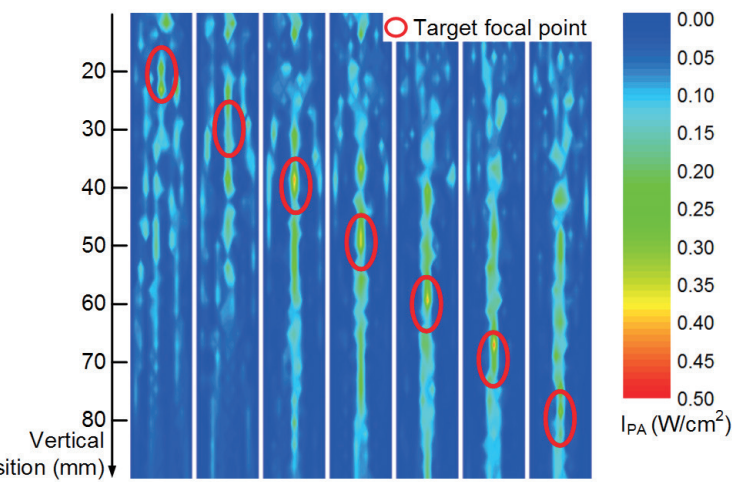

(b)

Fig. 9. (Color online) Spatial distributions of the pulse average ultrasound energy intensity $\left(I_{P A}\right)$ with the adjusted focal point in (a) horizontal and (b) vertical directions.

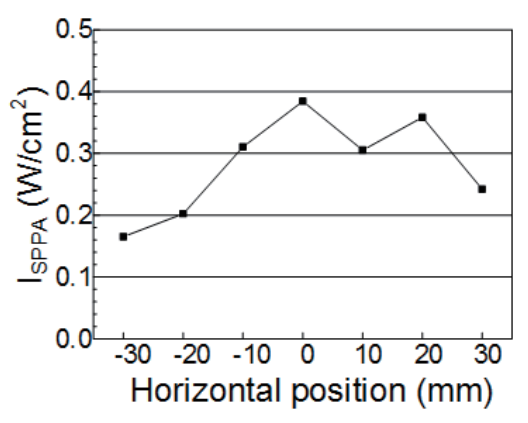

(a)

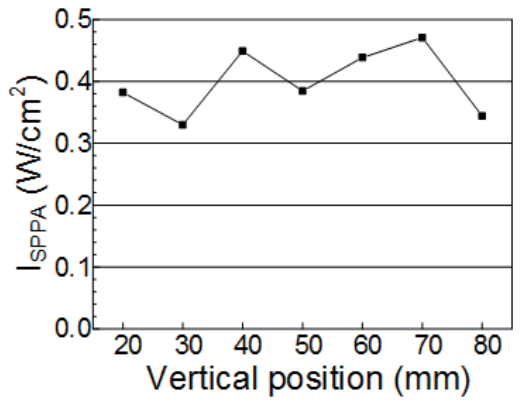

(b)

Fig. 10. Measured spatial peak-pulse average intensity $\left(I_{S P P A}\right)$ plotted as a function of the focal point location adjusted in (a) horizontal and (b) vertical directions.

as 1 to $5 \mathrm{~mm}$ in the vertical direction. The average targeting error in the vertical direction was calculated as $3.86 \mathrm{~mm}$. The radius of a focusing area, where the $I_{P A}$ is larger than half of the $I_{S P P A}$, was measured as approximately $1.05 \mathrm{~mm}$.

In Fig. 10, variations of the $I_{S P P A}$ are plotted as a function of the location of the target focal points. In the horizontal direction, the $I_{S P P A}$ was measured in the range of 0.17 to $0.38 \mathrm{~W} / \mathrm{cm}^{2}$. In the vertical direction, that range was 0.32 to $0.48 \mathrm{~W} / \mathrm{cm}^{2}$. When the focal point was adjusted in the horizontal direction, the highest energy intensity was observed at the center point. The $I_{\text {SPPA }}$ is less fluctuated with the vertically adjusted focal points than with the horizontally adjusted ones.

\section{Discussion}

\subsection{Spatial accuracy of focused ultrasound}

The results of the beamforming experiment indicate that the developed 32-channel LIFU stimulator can be used to focus the ultrasound energy on an arbitrary target point. In the experiment, 
the focal point was successfully adjusted in the horizontal and vertical directions depending on the sonication time delays that were calculated according to the beamforming algorithm. Attributed to the fine delay control with a unit time step of $6.67 \mathrm{~ns}$, the focal position could be delicately adjusted with nearly zero targeting errors. In particular, the extremely accurate targeting with zero targeting error was obtained with the horizontally adjusted focal points. The measured focusing area with the radius of $1.05 \mathrm{~mm}$ and the minimum focus shifting of $9.87 \mu \mathrm{m}$ are sufficiently small to provide high spatial resolution for targeting sub-centimeter-sized targets, such as a bundle of peripheral nerves and muscle fibers with a thickness less than $2.5 \mathrm{~mm}{ }^{(26)}$

\subsection{Performance variation according to focal point location}

The experimental results on the spatial distributions of the focused ultrasound energy indicate that the focusing performance of the developed stimulator can be varied according to the location of the target focal point. As shown in Fig. 10, the $I_{S P P A}$ is varied as a function of the location of the focal point. In particular, a large variation of $I_{S P P A}$ is observed with the horizontally adjusted focal point. The ratio of the maximum $I_{S P P A}$ to the minimum one is calculated as 2.33 in the horizontal direction. On the other hand, a relatively small variation of focused ultrasound energy is obtained in the vertical direction, with that ratio of 1.43 .

For a more quantitative analysis of the variation of the focusing performance according to the focal position, a beam nonuniformity ratio (BNR) that indicates the degree of focusing is calculated. The BNR is defined as the ratio of the $I_{S P P A}$ to the spatial average-pulse average intensity $\left(I_{S A P A}\right)$ in a unit-sized square area around the focal point. Note that a higher BNR indicates a higher degree of focusing. In Fig. 11, the BNR is plotted as a function of the target focal positions in the horizontal and vertical directions. A larger variation of BNR is observed in the horizontal direction than in the vertical direction. The ratio of the maximum BNR to the minimum one is 1.63 in the horizontal direction. That in the vertical direction is determined as 1.19. In the horizontal direction, the BNR is sharply decreased as the focal point is shifted to the side positions.

From these results, it can be postulated that the highly uniform focusing performance is achieved with the vertically adjusted focal points. However, when the focal point is adjusted in the horizontal direction, the focusing performance can be relatively widely varied according to the

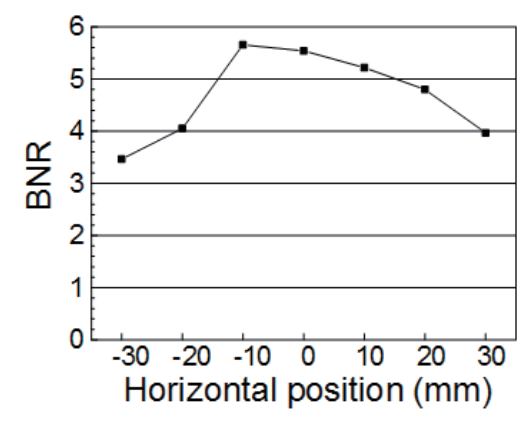

(a)

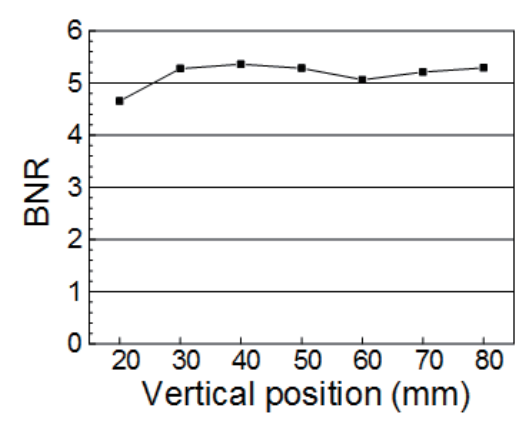

(b)

Fig. 11. Measured BNR plotted as a function of the focal point location adjusted in (a) horizontal and (b) vertical directions. 


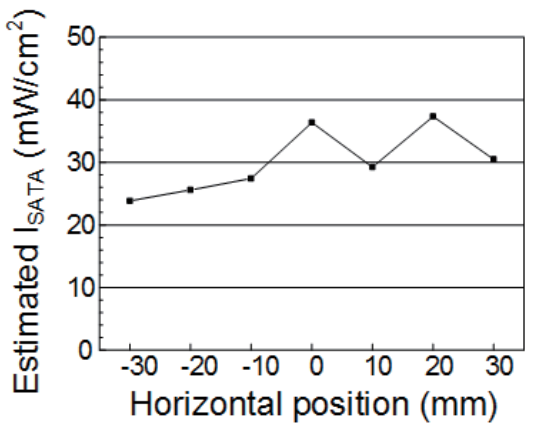

(a)

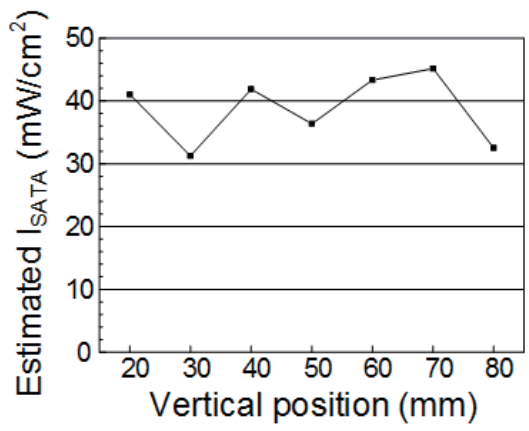

(b)

Fig. 12. Estimated spatial average-temporal average intensity $\left(I_{S A T A}\right)$ with the duty cycle of $50 \%$ plotted as a function of the focal point location adjusted in (a) horizontal and (b) vertical directions.

location of the focal point. In other words, the ultrasound energy is less propagated to the sidepositioned focal points because of the decreased beamforming efficiency. For compensating the degraded focusing performance in the side positions, additional compensation, such as the acoustic amplitude modification according to the location of the target focal points, should be provided.

\subsection{Potential for neuromuscular rehabilitation}

According to the previous study, the LIPUS with the spatial average-temporal average intensity $\left(I_{S A T A}\right)$ of $16 \mathrm{~mW} / \mathrm{cm}^{2}$ accelerated the peripheral nerve recovery. ${ }^{(9)}$ In other work, the LIPUS with the $I_{\text {SATA }}$ of $30 \mathrm{~mW} / \mathrm{cm}^{2}$ improved the regeneration of the injured muscle tissues. ${ }^{(13)}$ To deduce the developed system's potential for the neuromuscular rehabilitation, the $I_{S A T A}$ with the duty cycle of $50 \%$ is estimated from the measured $I_{S P P A}$ and BNR. In Fig. 12, the estimated $I_{S A T A}$ is plotted as a function of the target focal positions in the horizontal and vertical directions. The estimated $I_{\text {SATA }}$ is distributed in the range of 24 to $45 \mathrm{~mW} / \mathrm{cm}^{2}$. In addition, the energy intensity can be increased by more than 40 times because the driving voltage can be increased to $400 V_{P P}$. From this analysis, it can be postulated that the developed system can provide sufficient energy for the neuromuscular rehabilitation.

\section{Conclusions}

The 32-channel LIFU stimulator using phased array miniature PZT transducers is presented to provide highly accurate targeting in ultrasound-based neuromuscular rehabilitation. In the developed system, the beamforming technology is used to finely adjust the focal point without the geometrical shift and inconvenient replacement of the transducer. The experimental results show that the focal point can be delicately controlled in the horizontal and vertical directions. In addition, the improved targeting accuracy is provided with nearly zero targeting errors. The size of the focal point is sufficiently small to implement high-resolution targeting on peripheral nerves and a bundle of muscle fibers. Although the uniformity of focusing performance should be partially improved in the horizontal direction, it is expected that the developed stimulator can be effectively utilized to generate the accurately targeted LIFU stimulation for neuromuscular rehabilitation. 


\section{Acknowledgements}

The authors gratefully acknowledge the support of the Korea Health Technology R\&D Project through the Korea Health Industry Development Institute (KHIDI), funded by the Ministry of Health \& Welfare, Republic of Korea (HI14C3477), and of the Next-generation Medical Device Development Program for Newly Created Market of the National Research Foundation (NRF), funded by the Ministry of Science, ICT and Future Planning, Republic of Korea (2015M3D5A1066100). We also thank Dr. J. Park and Dr. C. Lee for their help in ultrasound measurements.

\section{References}

1 L. R. Sheffler and J. Chae: Muscle Nerve 35 (2007) 562.

2 B. M. Doucet, A. Lam, and L. Griffin: Yale J. Biol. Med. 85 (2012) 201.

3 P. H. Peckham and J. S. Knutson: Annu. Rev. Biomed. Eng. 7 (2005) 327.

4 D. N. Rushton: Disabil. Rehabil. 24 (2002) 407.

5 K. M. Bogie and R. J. Triolo: J. Rehabil. Res. Dev. 40 (2003) 469.

6 G. ter Haar: Eur J. Ultrasound 9 (1999) 3.

7 A. Bystritsky, A. S. Korb, P. K. Douglas, M. S. Cohen, W. P. Melega, A. P. Mulgaonkar, A. DeSalles, B. Min, and S. Yoo: Brain Stimul. 4 (2011) 125.

8 W. J. Tyler: The Neuroscientist 17 (2011) 25.

9 A. R. Crisci and A. L. Ferreira: Ultrasound Med. Biol. 28 (2002) 1335.

10 W. Jiang, Y. Wang, J. Tang, J. Peng, Y. Wang, Q. Guo, Z. Guo, P. Li, B. Xiao, and J. Zhang: Sci. Rep. 6 (2016) 22773.

11 C. Chang, S. Hsu, F. Lin, H. Chang, and C. Chang: J. Biomed. Mater. Res. B 75B (2005) 99.

12 H. Zhang, X. Lin, H. Wan, J. Li, and J. Li: Microsurgery 29 (2009) 479.

13 Y. Chan, K. Hsu, C. Kuo, S. Lee, S. Chen, W. Chen, and S. W. Ueng: Ultrasound Med. Biol. 36 (2010) 743.

14 K. Nagata, T. Nakamura, S. Fujihara, and E. Tanaka: Ann. Biomed. Eng. 41 (2013) 1095.

15 M. R. Bachtold, P. C. Rinaldi, J. P. Jones, F. Reines, and L. R. Price: Ultrasound Med. Biol. 24 (1998) 557.

16 V. Colucci, G. Strichartz, F. Jolesz, N. Vykhodtseva, and K. Hynynen: Ultrasound Med. Biol. 35 (2009) 1737.

17 B. Min, P. S. Yang, M. Bohlke, S. Park, D. R. Vago, T. J. Maher, and S. Yoo: J. Phys. Conf. Int. J. Imag. Syst. Tech. 21 (2011) 232.

18 S. Yoo, A. Bystritsky, J. Lee, Y. Zhang, K. Fischer, B. Min, N. J. McDannold, A. Pascual-Leone, and F. A. Jolesz: Neuroimage 56 (2011) 1267.

19 C. J. Wright, J. Rothwell, and N. Saffari: J. Phys. Conf. Ser. 581 (2015) 012003.

20 J. Lu, H. Zou, and J. F. Greenleaf: Ultrasound Med. Biol. 20 (1994) 403.

21 K. E. Thomenius: Proc. 1996 IEEE Ultrason. Symp., eds. M. Levy, S. C. Schneider, and B. R. McAvoy (IEEE, New York, 1996) pp. 1615-1622.

22 P. Kruizinga, F. Mastik, N. de Jong, A. F. W. van der Steen, and G. van Soest: IEEE Trans. Ultrason. Ferr. 59 (2012) 2684.

23 B. D. Steinberg: IEEE Trans. Ultrason. Ferr. 39 (1992) 716.

24 G. Gurun, J. S. Zahorian, A. Sisman, M. Karaman, P. E. Hasler, and F. L. Degertekin: IEEE Trans. Biomed. Circuits Syst. 6 (2012) 454.

25 H. Kim, A. Chiu, S. D. Lee, K. Fisher, and S. Yoo: Brain Stimul. 7 (2014) 748.

26 Q. Hogan: Anesthesiology 85 (1996) 37. 